\title{
The challenges in computer supported conceptual engineering design
}

Abstract: Computer Aided Engineering Design (CAED) supports the engineering design process during the detail design, but it is not commonly used in the conceptual design stage. This article explores through literature why this is and how the engineering design research community is responding through the development of new conceptual CAED systems and $\mathrm{HCl}$ (Human Computer Interface) prototypes. First the requirements and challenges for future conceptual CAED and $\mathrm{HCl}$ solutions to better support conceptual design are explored and categorised. Then the prototypes developed in both areas, since 2000, are discussed. Characteristics already considered and those required for future development of CAED systems and $\mathrm{HCls}$ are proposed and discussed, one of the key ones being experience. The prototypes reviewed offer innovative solutions, but only address selected requirements of conceptual design, and are thus unlikely to not provide a solution which would fit the wider needs of the engineering design industry. More importantly, while the majority of prototypes show promising results they are of low maturity and require further development.

\section{Introduction}

Current Computer Aided Engineering Design (CAED) tools are continually improving and those widely used for engineering design and manufacture in industry now successfully support and interlink detailed design, analysis, simulation and manufacturing (Fuge et al. 2012; Gao et al. 2000). Conceptual design is not typically performed in industry using CAED systems, and it is often claimed that this is due to a lack of support for conceptual design built into commercially used CAED systems (Verstijnen et al. 1998). Horváth (2000) finds that academic research on conceptual CAED has not been adopted by the industry, which is still happier to pay a designer to perform a conceptual design the way they find the most appropriate, than to invest in adoption of a more systemic solution. This paper reports a literature review exploring the reasons behind why current CAED systems are not used for conceptual design, what are the developments for conceptual CAED concerning human computer interface and software, and what the requirements and challenges for future conceptual CAED system development might be.

During the conceptual design stage, the design is changing frequently and evolving (Zhong et al. 2011), and the focus on detail is not as necessary as the need to generate and manipulate ideas quickly (Fuge et al. 2012). Designers create initial solutions, then modify or combine them and create concept variants to match the design requirements, placed on the product by either customers or the context of the product being designed (Müller et al. 2003; Zheng et al. 2001). Modelling using commercial 3D CAED systems requires concrete, precise and quantitative "design information as an input that is often not available at the conceptual design stage" (Zhong et al. 2011). At that stage specifications and constraints are often not fully established (Igwe et al. 2008). Designer's "attention is focused on the overall appearance of the model, and the exact dimensions, positions, tolerances, etc. are dealt with at later design stages" (Sharma et al. 2011). Design concepts are inherently uncertain and incomplete (Varga et al. 2007), and it is this ambiguity that can contribute to the development of design, often called design emergence (Evans 2005). This is why keeping design ideas vague, incomplete and sometimes even irrational, until they are sufficiently developed, is important during conceptual design (Company et al. 2009; Müller et al. 2003). CAED systems currently in use do not support intensive manipulation of graphical data to the degree required to enable this (Alcaide-Marzal et al. 2013; Shesh and Chen 2004).

Although a CAED system can be used for most design stages, the designer needs to adapt to the CAED system (Huang 2007). Complex interfaces used to interact with CAED systems are not suitable for early conceptual design (Fuge et al. 2012), as they lack seamless transition between activities and intuitive modes of interaction (Stark et al. 2010). The majority of commercially available CAED 
systems have a menu based WIMP (Window Icons Menus Pointer) interface (Sharma et al. 2011; Zhong et al. 2011). A WIMP interface requires extensive, often professional, training, due to the number of tools and procedures that need to be learned. A WIMP interface is referred to as traditional human computer interface in this paper. In terms of time, 3D CAED modelling has a steep learning curve and new users find the process of using the mouse and keyboard in a 2D plane to design in the 3D space tedious, lengthy and unintuitive (Dave et al. 2013; Gao et al. 2000; Zhong et al. 2011). Free-form spline modelling is used to design complex irregular shapes in CAED, and changes to splines require manipulation of splines via a large number of control vertices, which in spite of continuous development of CAED systems consumes large amounts of time and effort (IX et al. 2001). New mechanisms of interaction are required to make CAED systems easier to use (Rodriguez Esquivel et al. 2014). They need to be intuitive and incorporate natural human actions (Shankar and Rai 2014; Verma and Rai 2013; Ye et al. 2006). Alternative human computer interface ( $\mathrm{HCl})$ solutions, such as gesture based interface, VR (Virtual Reality) supported interfaces, and haptic interfaces are now being considered to obtain faster communication between the user and CAED systems (Esfahani and Sundararajan 2012).

Technical aspects, architecture of the CAED systems and functionality of interfaces are important, but so are the characteristics of the users who interact with them, experience being a key characteristic. It has been established that experience was required in order for a user to successfully employ currently commercially used CAED systems (Dadi et al. 2014). More recent CAED systems are developed in academia with the aim of being quickly mastered regardless of experience levels of the users, however the majority of these are still in experimental stages (Lawson 2005; Mayda and Börklü 2014). During CAED interface development user experience is considered largely in the interface evaluation stage, potentially leading to user sourced requirements for conceptual design not being captured.

This review will focus on state-of-the-art research undertaken to improve underlying architecture, procedures and workflows of CAED systems developed by academic researchers to support conceptual design and better integrate it with detailed design, analysis, simulation and manufacturing; and specific interfaces developed to improve interaction between the human and computer. By reviewing the literature exploring these two topics, we aim to identify the latest developments in computational support for the conceptual design stage in engineering design, and identify envisaged challenges.

Characteristics of conceptual design, and benefits and drawbacks of use of CAED for conceptual design, identified in the literature, are given in Sections 3 and 4. Requirements for CAED and interface development identified in the literature are presented in Section 5. The review of the recent developments in conceptual CAED software prototypes and $\mathrm{HCl}$ interfaces are given in Sections 6 and 7, respectively. Then a discussion is provided in Section 8 and conclusion in Section 9.

\section{Inclusion criteria}

Only papers published post 2000 were included in this review, similarly to what (Ferguson et al. 2014) did in their review, as the latest developments in technology and computing are a large contributor to the emerging CAED development for the conceptual design stage. While papers published before 2000 are just as likely to explore the topics this review is focusing on, the technology available to the authors would simply not be comparable, as the underlying computational technology has become both more powerful and less costly, and thus more attainable in the last 16 years.

Conceptual engineering design is the focus of the review, therefore major engineering databases were searched: Biotechnology and BioEngineering Abstracts (ProQuest), Compendex, Engineering Research Database (ProQuest), Science Direct, SCOPUS, Technology Research Database (ProQuest), and Web of Science. 
The papers included in the review have either presented new CAED system prototypes or new $\mathrm{HCl}$ interface prototypes, and they are marked with an asterisk in the list of references. To support the discussion behind the need for new conceptual CAED systems relevant papers discussing the related issues but not providing their own prototypes have also been reviewed. Papers discussing experience of users, and the effect it has on the use of CAED were also included.

Not all the papers identified focused on engineering design alone. Many primarily discuss architecture, automotive design and general design, but they were included, as in the early stages of design all three sectors encounter similar issues. Additionally to the best of the authors' knowledge, there are no widely commercially accepted solutions for conceptual design in any of the sectors, and successful prototypes could likely be used across them. Papers considering both form and function of the product being designed were included, as both are equally important for conceptual design in engineering. The majority of papers report on user interfaces for CAED systems, but those including gaming interfaces while implying the possibility of CAED application were included also. While the focus of the paper is CAED, systems similar to CAED were included too, in order to consider similar concepts. A graph illustrating the areas of research considered is presented in Figure 1.

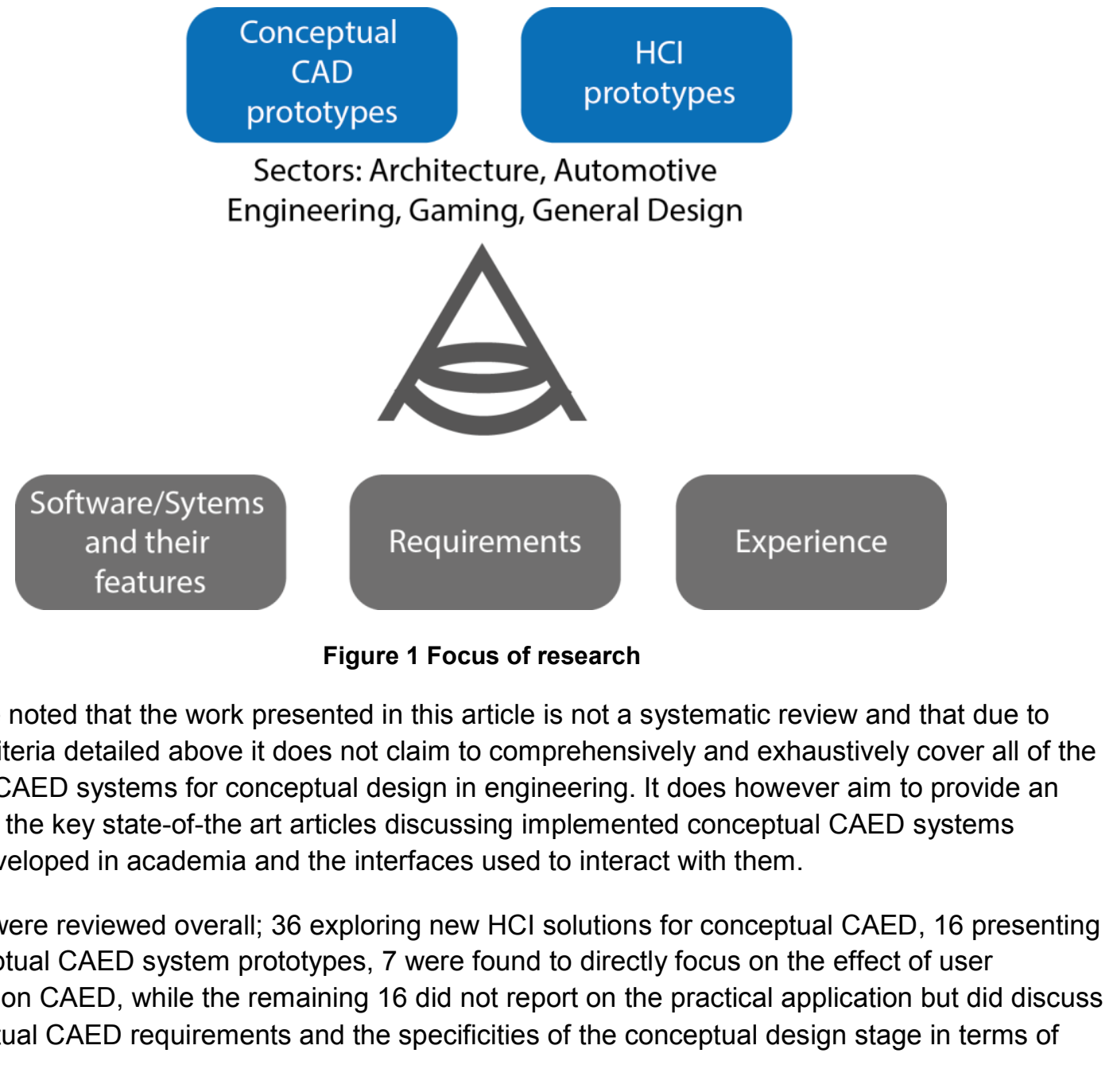

It should be noted that the work presented in this article is not a systematic review and that due to inclusion criteria detailed above it does not claim to comprehensively and exhaustively cover all of the aspects of CAED systems for conceptual design in engineering. It does however aim to provide an overview of the key state-of-the art articles discussing implemented conceptual CAED systems recently developed in academia and the interfaces used to interact with them.

75 papers were reviewed overall; 36 exploring new $\mathrm{HCl}$ solutions for conceptual CAED, 16 presenting new conceptual CAED system prototypes, 7 were found to directly focus on the effect of user experience on CAED, while the remaining 16 did not report on the practical application but did discuss the conceptual CAED requirements and the specificities of the conceptual design stage in terms of CAED.

\section{Types of conceptual design}

While discussing conceptual design authors often emphasise that it is important that it supports designers' creativity. In the context of engineering design, creativity is defined as a cognitive process or a problem solving process leading to the output/solutions/product that is both novel/unconventional and useful (Robertson and Radcliffe 2009), while satisfying certain requirements (Kryssanov et al. 
2001). Hanna and Barber (2001) add that the products created would be appraised by experts for their technical, social or aesthetic value. Creative problem solving makes unexpected connections between seemingly unrelated concepts (Lawson 2005; Robertson and Radcliffe 2009), and it is often said that this is due to the combination of two or more conceptual spaces (Bonnardel and Zenasni 2010), drawing ideas across domains (Kryssanov et al. 2001). During engineering problem solving it has been proposed that two knowledge representations interact - memories and images based on sensory impacts and experiences (known as perception based knowledge representations), and abstractions and generalisations of these experiences (known as meaning-based knowledge representations) (Robertson et al. 2007). They are considered to be a key aspect of creativity and so the creative process is often believed to be constructed of instances of abstraction and assignment of tangible form (Robertson et al. 2007).

Conceptual design and design ideation both occur early in the design process, and ideation can either precede the conceptual design or overlap with it. Design ideation has a specification of a desired function as an input, and a design concept specifies an achievement of the function as an output. Conceptual design has specification or functional requirements as an input, and produces one or a range of designs specifying a structure that meets the specification or the functional requirements as an output (Goel et al. 2012).

The conceptual design process can be observed from a variety of different standpoints. There is a generative phase in creative conceptual design where a mental representation is constructed, and an exploratory phase where its properties are interpreted (Shahin 2008). Designs can be adaptive designs (minor modifications to existing design - well suited for computers), development designs (existing design provides a starting point, but the design outcome differs from it significantly), or new designs (all stages are performed without an existing design used as an input) (Haik and Shahin 2010). Design can be routine (where the basic structure and the plans for the selection of parametric values are known), innovative (only the basic structure is known) or creative (the structure itself is unknown) (Goel et al. 2012). Considering the creative design strategies Li et al. (2007) split them into problem-oriented creative design (improve a product), function-oriented creative design (build a product to perform a specific function), product-oriented creative design (secondary function needs improvement) and form-oriented creative design (changing the shape and format). The conceptual CAED systems and $\mathrm{HCl}$ interfaces found in the literature and reviewed do not make a clear distinction between the types of conceptual design as they aim to provide support for the entire conceptual design process and all approaches to designs the users could take. It should be noted that this review will not make this distinction either, although it could be argued that depending on the key requirements a certain solution focuses on, in the future some solutions may be more suited to specific conceptual design variants. Conceptual engineering design could encompass all of the design varieties mentioned above.

Conceptual design is often performed as a group activity, and requires collaboration between different designers, integral for the creative process (Bermudez and Jones 2016). Designers can build on each other's knowledge and ideas, particularly in multidisciplinary design projects (Goel et al. 2012; Markopoulos et al. 2016). Shared understanding, or ability to collectively develop a design in the context of a project, with social interaction supporting the team cognition, is an important goal in early design stage collaboration (Gomes et al. 2016). Tools are being developed to support geographically distributed designers, working on shared projects while based in different locations (Bermudez and Jones 2016; Goel et al. 2012). Colocation has been found to help engage participants in design during building the shared understanding (Gomes et al. 2016), and digital tools can improvise this even when designers are geographically distributed.

\section{Benefits and drawbacks of the current CAED systems}

CAED is not used in conceptual design as much as it is used in detailed design, analysis, simulation and manufacture. Ways of expanding CAED to include the early stages of design have been 
considered. As already mentioned in the introduction, while CAED systems have developed greatly since their introduction, the WIMP interface is still the most commonly used interface, and it does not fully support conceptual design activities (Dickinson et al. 2005). Outside of CAED, sketching is the most common tool in conceptual design (Kokotovich and Purcell 2000). When 3D forms need to be created, designers sometimes prefer clay and foam modelling, as it helps them to resolve ergonomic features that are difficult to notice in digital form (Sener et al. 2002). Some designers find it essential to have a physical 3D model as soon as possible, allowing them to control and manipulate the form but avoid premature focus on detailed design, and instead include uncertainty and ambiguity that are missing from CAED (Evans 2005). If the conceptual design is performed independently of the CAED, sketching using pencil and paper or sketching software, the conceptual design outputs need to be reproduced in CAED to be further developed and eventually manufactured (Ye et al. 2006). The reproduction or conversion of sketches into CAED models is a time consuming and error-prone process (Dickinson et al. 2005), and the "lack of 3D geometric information in sketches and the imprecision associated with them makes them difficult to interpret algorithmically" and make the process automated (Shesh and Chen 2004). Creative activities associated with developing and communicating alternative solutions without much detail do not have the sequential structure CAED systems support (Igwe et al. 2008). In this section we summarise the findings from the literature exploring the benefits and drawbacks of CAED used for conceptual design, both CAED systems specifically designed for conceptual designs and instances where CAED intended for use in detailed design is used for conceptual design when appropriate.

\subsection{Benefits of CAED systems}

While the nature of CAED systems has changed over time, the reasoning behind their use has not they still tend to provide faster and more accurate solutions, and accurately capture detailed design information (Hartman, 2009). This is one of the key motivators to extend the CAED or a version of CAED to the conceptual design stage, and if fully achieved would be one of the key benefits they provide. Conceptual CAED is not commonly used in conceptual design stage in the industry, but academic research has explored it (Horváth 2000), and identified areas where conceptual CAED could bring significant benefits. Use of CAED in conceptual design introduces enhanced visualisation and communication, better group creativity and allows more time to be spent on ideas rather than detail, which are all positive influences (Robertson and Radcliffe 2009; Robertson et al. 2007). 3D sketching was found to "improve perception of visuo-spatial features, particularly in terms of unexpectedly discovering spatial features and relationships", and improving their problem finding behaviours (Rahimian and Ibrahim 2011). As conceptual design is often performed by teams of designers, ability of immersive tools to provide colocation and collaborative work in virtual environments, which are easily recorded and stored, holds potential significant advantage. Immersive virtual reality systems can help bring together conceptualisation and realisation, allowing designers to "experience" the designs (Horváth and Vroom 2015). Designers working in a team using VR in 3D sketching sessions have been found to maintain the same productivity, but perform more actions then using manual design procedures. Team work also leads to unexpected discovery of spatial features and relationships, and motivates the designers to share more ideas (Rahimian and Ibrahim 2011).

\subsection{Drawbacks of CAED systems}

Conceptual CAED developments hold potential for implementation, which supports the conceptual design process, and unifies it with the rest of the design stages. However, at the current stage of implementation and adoption there are many drawbacks tied to the use of CAED in conceptual design. Speed and accuracy of capture, which are the key motivation for the use of CAED in detailed engineering design, are questionable during the conceptual design stage, as WIMP lacks a variety of high level shape operators for designing and modifying model shapes which are required for conceptual design (Gao et al. 2000; Zheng et al. 2001). Customers may take part in evaluation of product design concepts in some domains, and it is difficult for customers to communicate their change intention for a product through commercially available CAED systems (Zhen-yu and Jian-rong 
2005). In some cases, design ideas can be influenced, and at times limited, by the capabilities of the software. The ideas are either not fully developed as they appear final earlier than they actually are, or the designs are not fully explored due to software limitations (Robertson and Radcliffe 2009).

Additionally, using a CAED system requires an interface for a designer to be able to input their ideas. Using a mouse and keyboard requires extra effort in planning the steps required to build the model, rather than form generation, and causes extra cognitive load and a mismatch between design thinking and command manipulation (Huang 2007). The technology behind representation of the 3D models in CAED systems makes modification of fine details of surfaces a difficult and time-consuming process (Gao and Gibson 2006). CAED is also found to be used prematurely, which leads to effects such as circumscribed thinking, premature fixation and bounded ideation (Veisz et al. 2012). Circumscribed thinking is when the design is limited by what it is possible to do with the tool, or even more limiting when the designers do what is easiest with the tools they have. Circumscribed thinking could also be "positive" - it prevents designers proficient in CAED from introducing unnecessary complexity and wasting resources (Musta'amal et al. 2008; Robertson and Radcliffe 2009; Robertson et al. 2007). Premature fixation happens when the CAED models become too detailed too early in the design process which discourages designers from making further changes (Robertson and Radcliffe 2009; Robertson et al. 2007). Bounded ideation is a consequence of designers focusing on CAED tools and how to use them rather than ideation, leading to less creative outputs (Robertson and Radcliffe 2009; Robertson et al. 2007).

One of the largest drawbacks of using a commercial CAED systems is the steep learning curve majority of systems have. Additionally, often the knowledge required is not intuitive and not necessarily closely linked to the design process one would use if paper sketch or clay modelling was used. According to Hartman (2009) CAED knowledge is declarative, procedural or strategic. Declarative knowledge, "knowledge that" (Chester 2007), is knowledge of commands in CAED, particular to a specific CAED system (Diwakaran and Johnson 2012; Hartman 2009). Procedural knowledge, "knowledge how" (Chester 2007), is knowledge of tools and processes (Hartman 2009), or the knowledge of possible alternate methods to create a CAED model (Diwakaran and Johnson 2012). It is not linked to any specific CAED system and this type of knowledge helps the experts perform better (Diwakaran and Johnson 2012). Strategic knowledge or strategic expertise, "metacognitive knowledge" (Chester 2007), includes knowledge of geometry creation, manipulation, and editing techniques coupled with information about the design considerations that surround the model creation process, software processes, and past experiences (Hartman 2009). According to Bhavnani et al. (1993) CAED expertise is differentiated by strategic and not command knowledge, and Bhavnani and John (1997) state that efficient use of design software is not guaranteed by good design or experience, but that it requires task decomposition strategies. Experts are able to solve problems more quickly and accurately than novices, due to their ability to put a problem in the specific context of a particular domain and strategic use of tools (Hartman 2009), and although they might take longer to create an original model it has a higher feature reuse as they consider the changes it might require in the future (Diwakaran and Johnson 2012).

Benefits and the drawbacks of CAED use are more or less prominent depending on the experience of the user. Experienced designers are less affected by circumscribed thinking, as they focus more on the requirements (Robertson and Radcliffe 2009; Robertson et al. 2007), but they are more likely to show mild levels of bounded ideation (Robertson and Radcliffe 2009; Robertson et al. 2007). Lawson (2005) claims that experienced designers use solution-focused rather than problem-focused strategies. Veisz et al. (2012) found that user experience affects their perception of CAED and that novice and experienced groups have very different perceptions about CAED capabilities. Musta'amal et al. (2008) found that CAED users can be classified as CAED recorders or CAED designers, the former perceiving the software as a tool for presentation of the final design ideas without involving them in idea creation, and the latter actively including the software during the design and using it for final idea representation. Different CAED users are at different points on the spectrum between these two extremes. 


\section{Requirements for conceptual CAED}

The requirements identified in the literature and summarised in this section cover the initial conceptual design activities and aim to allow creative and rapidly developed designs to be captured more efficiently. This should be supported by both suitable interface and CAED software structure that enables capture of the key design information, while allowing design review and evaluation. The requirements collated here were extracted from the papers reporting on developed CAED prototypes and developing $\mathrm{HCls}$ to control the conceptual CAED software, and it is noticeable that the key requirements identified by different authors focus on different aspects of the conceptual design process. Whether authors have attempted development of conceptual CAED systems or $\mathrm{HCl}$ for conceptual CAED, first they identified what needs to be improved, and how CAED systems could be changed or redesigned to offer better support for conceptual design. These requirements, sorted sequentially to match the stages of conceptual design process, are presented in this section. They can be clustered into three groups shown in Figure 2.

Initial

Ideation Process
Digitisation of design / translation of design
Design review

\& evaluation

Figure 2 Requirement groupings

\subsection{Initial ideation process requirements}

The first cluster of requirements explores the kind of environments that should be created to support conceptual design and how ambiguity characterising it could be maintained. To support initial ideation process it is necessary to:

- Introduce minimal disruption to designers work process (Krish 2011), as the creation of novel ideas requires smooth flow of thoughts (Dickinson et al. 2005).

- Have representation means that are in harmony with the speed of ideation and natural thinking (Dickinson et al. 2005; Horváth 2000) - the design needs to be visualised as it is being carried out (Vidal and Mulet 2006).

- Have tools that are not an obstacle to creativity but that could stimulate creativity (Krish 2011; Vidal and Mulet 2006).

- Have features that remind the user sketching is tentative not definitive (Company et al. 2009), and which tolerate ambiguity and incompleteness (Oh et al. 2006).

- Have features that keep the advantages of sketching and avoid weaknesses (Company et al. 2009), combine support for creativity (the wide range of information sketches can contain, from spatial to tentative temporary lines, motion lines, symbols etc.) with the advantages of digital system environments (visualisation) (Müller et al. 2003).

Majority of these requirements are not tool specific and indicate existing misalignment between the capabilities of currently available tools and the pace and nature of the conceptual design process. New developments should refrain from interrupting the design process, as that often inhibits it, and would need to be able to provide sufficiently fast response from tools, and intuitive user interfaces. The challenge to achieve ambiguous and tentative designs, which can then be transposed to detail design without design rework, is tied to data management issues and is a major challenge that is partially decomposed through the next cluster of requirements given in this section. Finally, there is the desire to maintain the beneficial aspects of traditional conceptual design techniques such as sketching, but adapted to the advancing technology.

\subsection{Digitising of design/translation of design requirements}

The second cluster of requirements focuses on the kind of support designers require while using the software and externalising their ideas by inputting them into a CAED software giving them a digital form. These requirements explore the nature of CAED software and ways to successfully adapt it to 
conceptual design. To digitise design or transformation of design ideas into digital conceptual "sketches" it is necessary to:

- Provide assistance with repetitive tasks (e.g. symmetry, drawing all edges in extrusion like objects) (Company et al. 2009).

- Have CAED software with as non-intrusive an interface as possible (Oh et al. 2006), and allow the designer to focus on the design rather than the system interface, procedures or interaction constraints (Fiorentino et al. 2010; Ye et al. 2006).

- Maintain the characteristics of object interaction that would exist during the development of new modelling systems, while using the advantages of computer simulation (Dave et al. 2013).

- Allow human input when needed/wanted, and assist the human designer but not interfere with their thinking processes (Krish 2011; Shahin 2008).

- Provide knowledge management support to design thinking (Lawson 2005; Vidal and Mulet 2006), provide cognitive artefacts for designers without experience (Dorta et al. 2008), advice on what, how and when to reuse from previous experience (Kryssanov et al. 2001), and observe computer as learner, informer, critic, collaborator and initiator agent (Lawson 2005). The alternative approach is having a system capable of creative and innovative thinking/processing (Shahin 2008).

- Represent a range of levels of abstraction (Israel et al. 2009; Oh et al. 2006).Vague concepts need to be represented as well as detailed ones (Krish 2011).

- Allow collaborative work (Vidal and Mulet 2006).

The second cluster of requirements focuses on different types of computer support being developed. The appropriate implementation of knowledge support or computer generated designs based on prior knowledge which could serve as inspiration would be one possible route. Ability to represent different levels of abstraction as a part of the same project is similar to the requirement to maintain ambiguity presented in the first cluster, but here given in terms of CAED tools requirements. Finally, CAED tools should bring a number of benefits stemming from the ability of computers to speed up processes which can be mathematically defined such as symmetry or multiplication, and the ability to allow collaborative work, co-located or distributed, as team work is one of the characteristics of the conceptual design.

\subsection{Design review and evaluation requirements}

As evaluation is an integral part of conceptual design it is essential for conceptual CAED systems, and $\mathrm{HCl}$ used to control them, to support design review and evaluation by:

- Integrating conceptual CAED with the subsequent phases of the design process (CAED, CAE, CAM etc.) (Company et al. 2009) by providing consistency between concept, design, and manufacturing (Brunetti and Golob 2000). Conceptual design outputs need to be able to be used as the input for further CAED based activities (Horváth 2000) and enable an efficient transition to detailed design (Krish 2011).

- Having the ability to hold several versions in parallel, representing them efficiently, and combining their elements (Krish 2011; Lawson 2005).

- Having the ability to revisit a particular design's history - ways of storing the creative concepts at different levels of development and allowing review (Israel et al. 2009).

- Providing different views for different types of representation (Company et al. 2009).

- Considering design intent in design exploration as it provides crucial information about the design rationale (Shankar and Rai 2014; Verma and Rai 2013).

- Providing support specifically for evaluation (Oh et al. 2006).

Majority of review and evaluation based requirements can be summarised as a higher level requirement to integrate all stages of the design process, and ensure data and knowledge integration for the complete design, including all of the development stages, to be easily accessible. This would also require appropriate representation is available for all types of data. 


\section{Conceptual CAED system prototypes}

In the papers reviewed 16 prototypes were developed. Some of them are based on the use of new displays (stereoscopic glasses or VR-helmets, monitor-based 3D display that directly tracks a single user's eyes and dynamically tweaks the image to achieve 3D effects, spherical 3D display that renders coloured volumetric elements that can be viewed from any angle) or new interaction technologies (3D mice, electronic pens, tablets with and tablets without digital screens) (Dickinson et al. 2005). Others focus on meeting the requirements derived from the design process and design models. For example almost all techniques embed a certain level of abstraction, which then inevitably means that during embodiment or detailing further information or effort is required from the designer. While computers are good at automating processes, conceptual design usually has too many varied parameters, and too little information embedded to be automatised successfully (Horváth 2000), therefore different approaches have been explored to overcome this issue by allowing the users a different kind of information input.

It should be noted that there was a limited amount of information available, in the articles reviewed, providing comparison of effectiveness of the systems developed and traditional CAED systems, or effectiveness of systems developed and analogue conceptual design approaches. The same applies to the HCls described in Section 7. Additionally, due to the vastly different approaches to conceptual design support between different prototypes, and $\mathrm{HCls}$ developed for different systems, it was impossible to compare them quantitatively, and we have instead categorised them based on their focus and capabilities.

Observing the conceptual CAED software prototypes we have found, based on the key approach to conceptual design they take, they generally fall under one of five categories:

- CAED relying on data capture

- Knowledge based CAED or CAED providing knowledge support

- Functional CAED

- CAED approaches emulating or improving traditional sketching

- Feature based CAED

As shown in Figure 3, all but one of the reviewed CAED prototypes focused on a single one of these categories in their approach to providing a new conceptual CAED system. The five inner circles in Figure 3 mark the category the CAED software developed belongs to, and they are colour coded for easier differentiation. They are ordered from less specific to more specific, with regards to key design activity performed using the software. The outer circle displays more information on each individual solution, in terms of specific techniques used or specific actions supported by the new conceptual CAED system. 


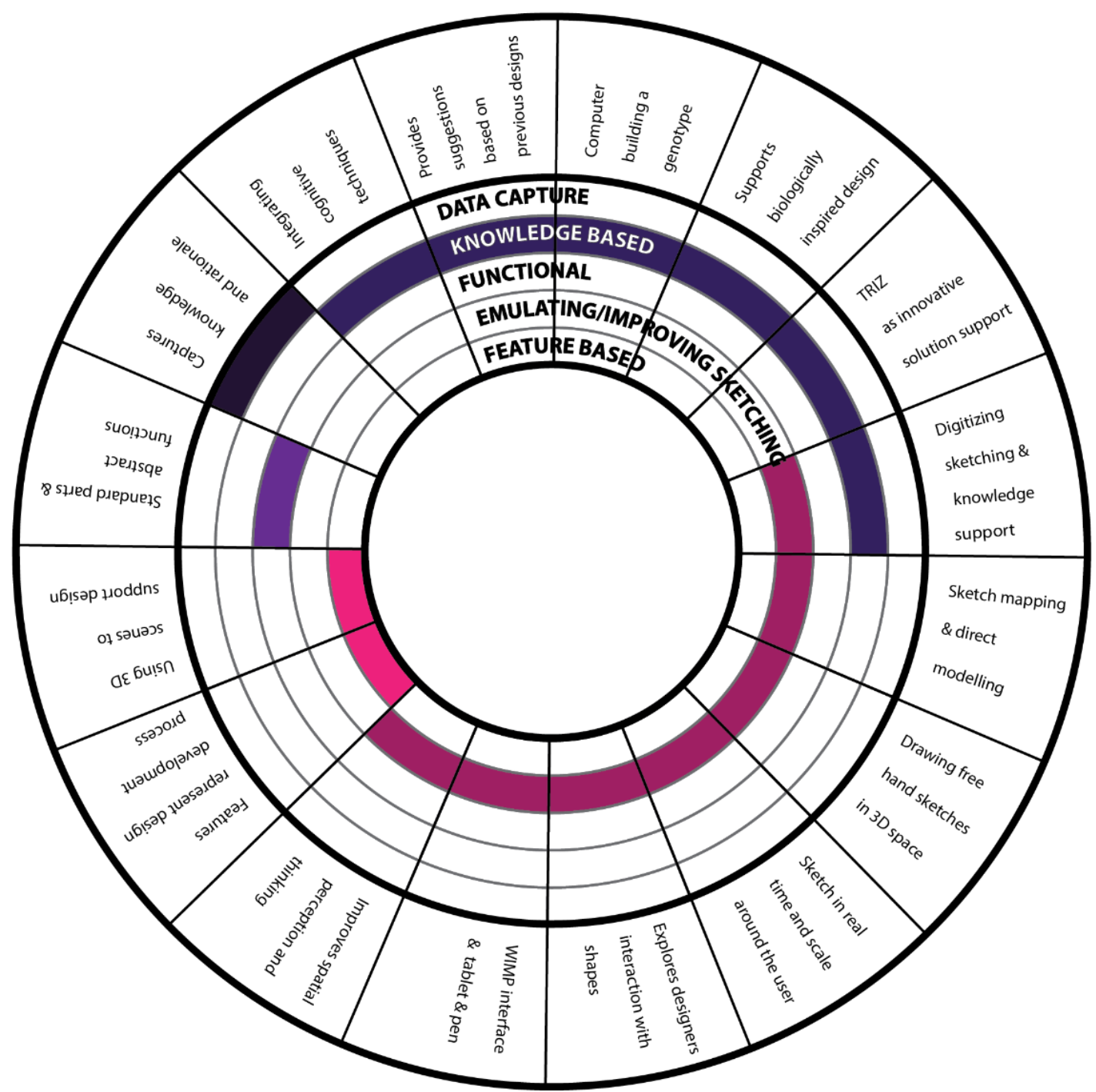

Figure 3 CAED system prototype overview

One CAED system was based on data capture. Sivanathan et al. (2015) developed and tested a framework which aimed to capture designers' knowledge and rationale throughout the design process, paying particular attention to the high levels of abstraction required in conceptual design.

The majority of knowledge based CAED systems that support conceptual design have focused on allowing the user to control the dynamics of interaction with the system (Bonnardel and Zenasni 2010; Goel et al. 2012; Li et al. 2007; Shahin 2008). Two solutions allowed the system to generate a design, and then allowed the users to modify it (Krish 2011; Mayda and Börklü 2014). They had knowledge bases or database management systems collating previous solutions or in some cases suggesting biologically inspired designs. Biologically inspired designs have been popular in architecture, and some researchers believe they would be suitable for mechanical engineering. Some solutions kept track of users' activities and suggested changes or ideas to augment the design (Li et al. 2007; Shahin 2008). Some include an FBS (Function-Behaviour-Structure) approach (Goel et al. 2012; Li et al. 2007) and some use TRIZ (Li et al. 2007; Mayda and Börklü 2014) to support ideation, and attempt to guide a designer through a systematic process. Shahin (2008) believes organising the information in a systematic way means that designers can focus on creative activities. Most have been developed as a proof of concept, rather than software for commercial use. 
One CAED system prototype was based on function. Bruno et al. (2003) focused on the functional representation of the problem in conceptual design, taking the functional dataset through different levels of abstractions. As sketching is seen as a quintessential conceptual design activity, a number of CAED systems have attempted to adapt it to the digital environment. Even where sketching may not be necessary for the generation of designs, externalising an idea can serve a communicative role (Bilda, 2005). Some prototypes used the tablet as a digital sketchbook and digitalised 2D sketching, additionally providing knowledge support during sketching (Hoeben and Stappers 2005). Others provided the option of drawing 2D sketches on 3D surfaces and thus mapping the sketches onto the 3D models (Tovey and Owen 2000). A step further was provided by prototypes allowing 2D sketching using software that automatically recognised the shapes and converted them into 3D models (Company et al. 2009; Jowers et al. 2008). 3D sketching in 3D space was also explored (Dorta et al. 2008; Israel et al. 2009; Müller et al. 2003). All of these approaches have been found to show potential, but all also require further development as none of the systems were at the point where they could be used commercially. Most sketch based CAED systems reviewed were developed for automotive applications, as they require designs comprised of geometrically irregular shapes.

Feature based solutions endeavour to retain the current CAED systems and expand them with new capabilities. They preserve the key characteristics of the interface and include new functions required to support the conceptual design. Two feature based prototypes were identified. One employed sketch recognition and transformed sketches into solids, with the visualisation dependant on the level of abstraction needed (Oh et al. 2006). The other focussed on data and attempted to retain all the information about the design throughout different levels of abstraction supported by different feature representations (Brunetti and Golob 2000).

\section{Human computer interface for conceptual CAED systems}

New conceptual CAED systems still require interaction tools, and a variety of different types of interfaces are being developed to control CAED software, input, modification, manipulation and visualisation of data. Five categories of specific actions performed in different systems discussed in the reviewed papers were derived through observation, and include (ordered from less specific to more specific, with regards to key design activity performed using the interfaces):

- Manipulation - Rotation, translation, zooming; changing the viewpoint without actually changing the model.

- Modification - Changing the model e.g. subtraction, addition, change of shape.

- Digital Sculpting/Surface Modification - Modification of shape but applied through surface modification, often emulating traditional sculpting.

- 3D Sketching - Sketching in the 3D environment. Either full 3D sketching or 2D sketching that is then used as a basis to extrude into a 3D shape, or a mix of both.

- Feature Modelling - Adding and subtracting features similar to traditional CAED systems.

Technology developments have enabled attempts to control CAED systems using interfaces not typically associated with them. In this section we review them grouped by the type of interface. Most of the interfaces developed are human centric, with a trend towards the exclusion of wearable devices (Lee et al. 2013). Use of touchscreens for CAED input is explored in Section 7.1. Haptic interfaces are explored as a stand-alone interface presented in Section 7.2, but are also included in some of the multimodal interfaces presented in Section 7.5. As pen and paper are still a dominant mode of sketching, and sketching is commonly used in conceptual design, researchers also explore how pen input can be used for CAED application discussed in Section 7.3. The newest and the least mature approach is the use of brain computer interfaces, allowing users to control CAED using their brain activity - these are presented in section 7.4. An increasingly popular approach is the use of multimodal interfaces. These combine a variety of different interfaces in the same solution, and can include gesture based interface, motion capture, gaze capture, haptic interface, virtual reality, tablet, mouse, pen, speech and virtual reality. Three specific interface sub-groupings were encountered 
repeatedly in different sources, and these are reported on in Section 7.5. A number of interfaces that did not fall into any of the groups listed above, but are relevant to CAED interface development, are reviewed in Section 7.6.

An overview of the $\mathrm{HCl}$ solutions, technology they employ and actions those technologies enable is given in Figure 4. This figure can be referred to when reading Sections 7.1 through 7.6, to provide context for the technologies discussed. The five inner circles in Figure 3 mark the category the CAED software developed belongs to/activities it supports, and they are colour coded for easier review. They are ordered from less specific to more specific. The black circle and lines group them according to the type of interface they employ, and the interfaces are not ordered in any specific way, except to keep the same types of interface grouped together. The outer circles display more information on each individual solution, in terms of specific technologies used.

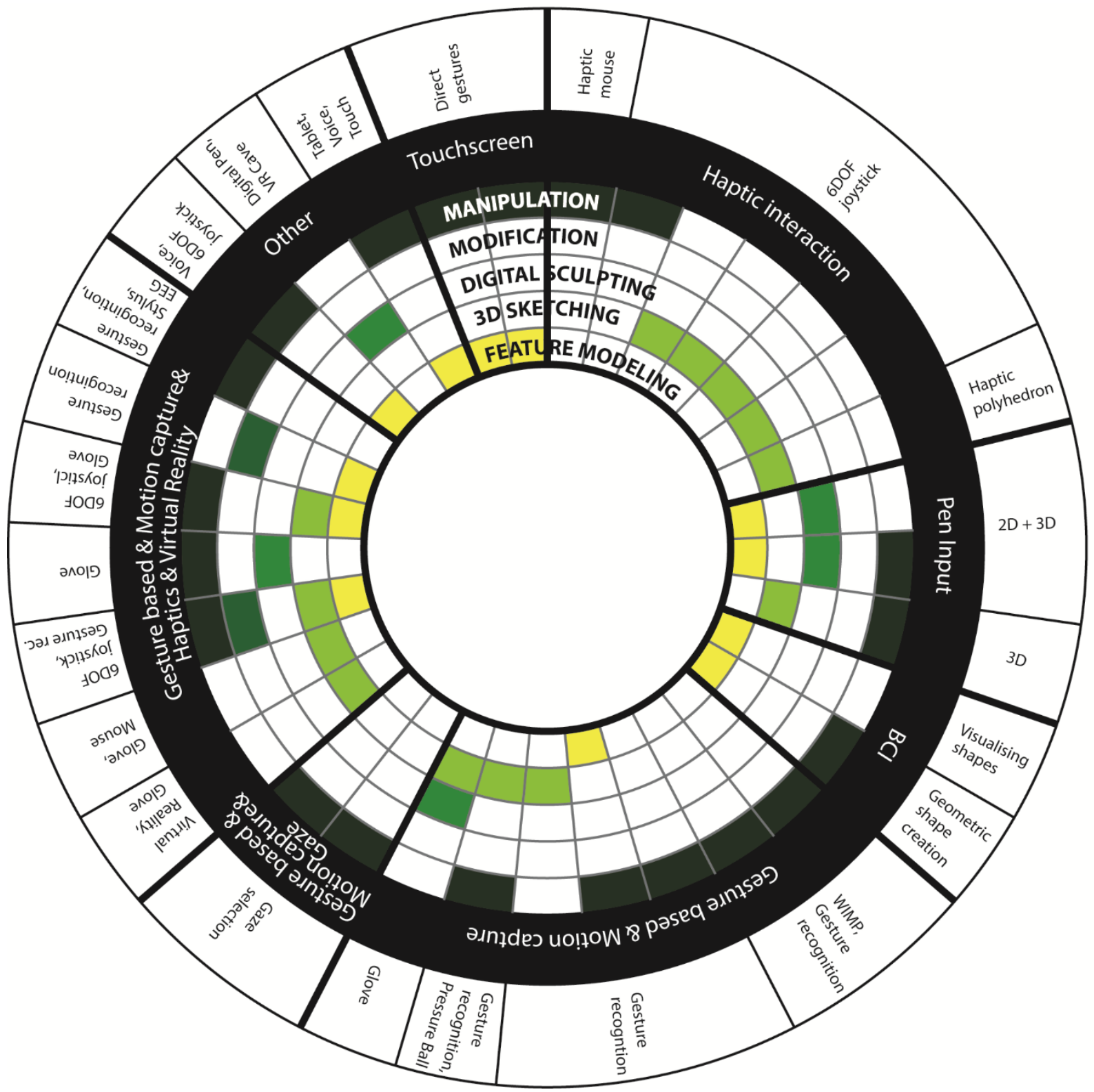

Figure 4 Overview of $\mathrm{HCls}$, technologies enabling them and the activities they support 


\subsection{Touchscreen}

Touch based interfaces are those in which designers use their fingers on the touch screen of a device to input design information. The technology is now omnipresent and easily attainable, and the question has arisen of whether CAED software could be used on them.

Kang et al. (2015) introduced a system where models were generated on a "smart" device, then transferred, in an editable form, to a PC system for detailed modelling. Once finished the model could not be edited on a smart device. The models were also not fully dimensioned and could not support addition or modification of constraints. Gestures used to input the data can be direct or symbolic, with direct gestures are currently preferred in the research community (Radhakrishnan et al. 2013).

While touch based input appears to be intuitive, it has certain drawbacks. It is not as precise as a mouse, finger occlusion of the screen occurs (Radhakrishnan et al. 2013), and complex or precise tasks are hard to perform on a small screen (Kang et al. 2015). The mouse outperforms the touch interface in both completion time and error levels (Radhakrishnan et al. 2013). 3D CAED systems also require high-end computational resources, which smart devices still do not provide (Kang et al., 2015). Additionally it has been reported that the optimal number of gestures and functions, and the impact of direct and symbolic gestures for touchscreens should be further researched (Kang et al. 2015; Radhakrishnan et al. 2013).

\subsection{Haptic Interface}

Haptics, devices that rely on applying tactile sensation and control in order to interact with computer applications, have been in development over the last two decades (Sener et al. 2002). They support natural communication between a human and a computer requiring the provision of minimal numerical detail about the design (Igwe et al. 2008). They are seen to have high potential to enable both individual and collaborative conceptual design (Bermudez and Jones 2016). Historically designers have used clay to make 3D physical models in order to explore different concepts (Igwe et al. 2008). Haptics allow them to simulate clay modelling tools, and sometimes this process is called "virtual clay" (Sener et al. 2002). They are used as input devices, but also for virtual assembly and other types of interaction with the models (Kyung et al. 2006). They improve working efficiency of CAD/CAM systems (Zhu 2008), and could potentially shorten the product development cycle due to being more user friendly, and more intuitive and easier to use, even if the users are not professional designers (IX et al. 2001). Haptics allow force feedback and more accurate tactile stimulation of surface properties of a virtual object (Kyung et al. 2006). Haptics aid the design process by conveying a virtual reality to humans more realistically, allowing faster design and free form design more appropriate for irregular shapes, and aiding the assembly process through allowing the user to feel the contact force (Kyung et al. 2006; Liu et al. 2005; Zhu 2008). They also enable deformation of CAED surface models in real time by pushing, pulling and dragging surfaces, making the design of complex surface models easier and faster than with traditional 2D interfaces (Liu et al. 2005).

Zhu (2008) developed a methodology for haptic interaction with CAED models in commercially available CAED software. Evans (2005) compared the foam based sketch modelling and virtual techniques supported by a haptic interface. Using haptics has proven to show high levels of creativity in the final product, not often noticed while using other modelling techniques (Evans, 2005). While haptics generally employ a 6DOF ( 6 degrees of freedom) joystick, different tools have also been used. A shape on the screen (a sphere) had been modified by changing the shape of the haptic polyhedron in reality, performing finer and more complex designs than with conventional haptics (Ogawa et al. 2006). Gao and Gibson (2006) introduced a tool-model haptic interaction, which while still using a 6DOF joystick, allowed a tool of a specific shape to be used to model a design solution, leaving indentations in the model in the shape of the tool. Both the tool and the shape having been designed had material properties, and elastic and plastic deformation were simulated. IX et al. (2001) enriched the haptic interfaces, so that in addition to force feedback they displayed modification in a 
physically plausible manner, and planned to include temperature in the future (hotter regions would deform more easily, colder would be stiff).

While haptics lend themselves to irregular shape creation and modification with successful results, if feature operation is required with regular shapes (e.g. creating a square or drawing a circle) they would not be the best choice, as creation of these shapes using free hand haptic devices is difficult. Therefore haptics tend to be an add on to already existing interfaces (Liu et al. 2005). Fine surface definition had proven similarly difficult when using haptics (Evans, 2005). The mainstream industrial applicability of the haptic solutions has been questioned (Evans 2005).

\subsection{Pen input}

Three papers were identified where the initial data input used a digital pen combined with a graphical tablet. They were used to sketch in $2 \mathrm{D}$ or $3 \mathrm{D}$, sketch on 3D models, or sketch in $2 \mathrm{D}$ where the software recognised shapes and extruded them into 3D solids.

Alcaide-Marzal et al. (2013) attempted digital sculpting using polygonal meshes, but the initial strokes were performed using a pen and a graphics tablet. The material was then added or removed based on a sketch and 3D shapes are constructed this way. Kim and Kim (2006) devised a modelling environment where pen markings were segmented to enhance the recognition and then primitive shapes were inferred. Manipulation could be immediate, employing 3D modelling operations (e.g. extrusions, transformations), or drawing operations (e.g. line and curve primitives). Shesh and Chen (2004) presented Smartpaper - a sketching environment supporting both 2D and sketching directly on a 3D model, with the emphasis on direct sketching but also supporting gestured sketching. The environment supported casual sketching - unsteady, discontinuous, overlapping strokes, implemented on a tablet PC. It could sketch a 3D object, but it could also extrude sketches (feature modelling), cut features using sketch profiles, join two objects and recognize gestures for drawing and cutting.

All three interfaces were in the early stages of application (for example the system developed by Shesh and Chen (2004) only supports drawn edges that are straight lines), but showed promise at the time and researchers were hoping to explore ways to produce more effective work flows for the pen input based interfaces in the future. Times required to learn how to use these interfaces and time spent performing tasks using them were comparable or sometimes even shorter than those in the conventional commercially available CAED systems (Kim and Kim 2006).

\subsection{Brain-Computer Interfaces}

Brain-Computer Interfaces $(\mathrm{BCl})$ are the latest development in human computer interfaces, and they use EMG (Electromyography) and/or EEG (Electroencephalography) signals for design information input, either by feature modelling or imagining shapes. Two studies exploring each will be detailed in this section. The interface is based on brain wave detection only or complimented with facial expressions detection, the latter being more efficient. As EMG and EEG activity can be recorded from the surface of the scalp/skin, respectively, the advancement of this approach can be attributed to its non-invasive nature (Verma and Rai 2013).

Shankar and Rai (2014) reported a study in two papers that uses Google SketchUp software as a platform to test the interface. It used the Emotiv headset which detects EMG and EEG signals and layed out a preliminary foundation for the use of BCI capability for 3D CAED modelling (Shankar and Rai, 2014). CAED models were constructed based on three operations: geometric shape creation, model editing by resizing or geometric operations such as booleans, sweeps and extrusions, and model rotation and translation. Modelling began with a 2D sketch using line or arc tool (position of the cursor governed by head movements), and then the 2D sketch was extruded (extrusion associated with thoughts "push" and "pull") to produce the final 3D part. Other functions had relied on electrical 
activity resulting from muscular movements during generation of facial expressions (EMG) (Verma and Rai 2013). The headset system needed to be trained to recognize user specific EMG/EEG signals (Shankar and Rai 2014), but the experiments have demonstrated that users get used to the interface relatively quickly (tasks of the same level of difficulty were performed more effectively in the second run) (Verma and Rai 2013). However, the participants did experience fatigue (Shankar and Rai 2014). Individual participant performances were statistically similar indicating the potential for $\mathrm{BCl}$ as a generalised CAED modelling medium that would not be significantly affected by individual differences between the designers (Verma and Rai 2013).

Esfahani and Sundararajan (2012) experimented with training a classifier to distinguish between primitive shapes (cube, sphere, cylinder, pyramid, or cone) imagined by a user, based on their associated EEG activity. The average accuracy of shape recognition from EEG activity following training was $44.6 \%$, significantly above chance level of $20 \%$. Visual imagery could be object based (shapes or colours) or spatial (location and spatial relations), and it consists of image generation (imagining a shape) and image maintenance (keeping that shape in memory).

$\mathrm{BCl}$ are in very early stages of implementation in a design context, and although they show promise there are still no common approaches to their development, clear guidelines for future development or fully functional proofs of concept. These $\mathrm{BCl}$ approaches also require lengthy calibration sessions before each use, and their accuracy is limited, thereby restricting their practical applicability.

\subsection{Multimodal gesture based interface}

Hand gestures are a natural, expressive and frequently used mode of human interaction (Tumkor et al. 2013). Gestural interfaces aim to be highly intuitive and offer free form modelling systems that emulate interaction with physical products (Dave et al. 2013), allowing designers to create 3D conceptual models quickly, while possessing a minimal amount of experience (Zhong et al. 2011). Approaches using gestures translated into CAED commands using motion capture will be covered in this section. They are further supported by haptic interface, gaze capture, or virtual reality in different combinations in order to provide a more natural input solution for CAED. In the literature reviewed three different groupings are most commonly made:

- Gesture based interface and motion capture

- Gesture based interface, motion capture and gaze

- Gesture based interface, motion capture, and /or haptic interface and/or virtual reality

\subsubsection{Gesture and motion capture based interface}

While the main goal is similar, different researchers have taken different approaches to providing an intuitive gesture based interface. Rodriguez Esquivel et al. (2014) explored ways to identify a series of gestures suitable for control of a CAED system. Tumkor et al. (2013) attempted to create a gestural system which integrates itself with existing GUI (graphical user interface) for CAED. They have also developed special menus for CAED software to support gesture based interface, which increased users' performance in comparison with the conventional menus. They found that in order to enable the use of hand gestures, fundamental changes in user interface were necessary and expected to be designed in the future. These systems could also allow users to simultaneously and collaboratively design using contactless gesture systems, with a higher ease of use than a mouse (Tumkor et al. 2013). Most approaches were based on the motion capture of hand movements, often using cameras. Marker-less gesture recognition (gesture recognition that does not require markers to be placed on users hands/gloves in order for the movement to be captured by the motion capture system) was the goal, as users often dislike wearable devices (Lee et al. 2013). Microsoft Kinect depth cameras have often been used for this purpose (Tumkor et al. 2013). Fuge et al. (2012) demonstrated the hand gesture based modelling approach supporting intuitive input, however they used gloves, headset and optical tracking. Wang et al. (2011) propose the use of gesture input that is only used where it improves the design process, which can fall back on mouse and keyboard whenever needed. One of 
the gesture recognition approaches was to mimic the process of traditional clay modelling and capture users' intent in a way that allowed them to communicate their intent to the system intuitively (Dave et al. 2013). Huang (2007) used a different virtual clay approach, they have, instead of capturing hand movements, developed and assessed an interface in which one hand is used to rotate the object while the other holds and squeezes a ball, which depending on the level of pressure creates different sized ink dots creating 3D forms. A camera was still used to support the spatial location.

Most studies only tested the feasibility of the interface development, and not the user experience (Fuge et al. 2012). Those that did test user perception found the new interfaces to be intuitive, but cause fatigue. Also it had been apparent that there was a learning curve to the systems (Bonnardel and Zenasni 2010; Dave et al. 2013). Commonality between the studies was that the gestures used were prescribed, which simplified the technology implementation but reduced user satisfaction. It is likely that humans are only able to store seven plus or minus two elements in their short term memory (Miller 1956), and this might be a factor when the gestures are prescribed.

\subsubsection{Gesture, motion capture and gaze based interface}

The second group of multimodal interfaces adds gaze capture to the list of the input mechanisms. Lee et al. (2013) tested this in a game environment but believed a similar approach could work for CAED. Song et al. (2014) developed an intuitive interface GaFinC, which combined hand gestures and gaze. Both used gaze to select an object which an action would be performed on. The former used remote cameras to detect the gaze and Kinect cameras for gesture recognition. The latter used a customized head mounted type gaze tracker and two Kinect depth cameras for gesture detection.

In these interfaces the remote cameras had some issues with different cornea recognition, but they also had the benefit of provision of human centric interface without a wearable device. Although gaze recognition is more intuitive than keyboard and mouse it is still more time consuming to operate. Additionally, wearing a mounted head set was not as comfortable as using the mouse.

\subsubsection{Gesture, motion capture, haptic and virtual reality based interface}

A more immersive design process was attempted by combining the gesture and motion capture described earlier in a VR environment, sometimes extending it to include haptics for better interaction with the system. VR allows more rapid, straightforward and natural approach to design creation and evaluation (Ye et al. 2006). Haptics were considered in order to allow a more realistic feeling of the shape for users during sculpting (Igwe et al. 2008).

Some authors focused on collaborative part design and evaluation (Bourdot et al. 2010). Precision manipulations were not a priority in these systems, intuitiveness and natural interaction were (Naef and Payne 2007). Often they had a strong evaluation focus, and aimed to provide the option for design review and modification of designs by non-expert customers (Kavakli et al. 2007; Naef and Payne 2007; Zhen-yu and Jian-rong 2005). Asadzadeh et al. (2012) explored a solution using clothes integrated RFID (Radio-frequency identification) which allowed users to interact with large displays in public collaboration spaces without the need to carry a dedicated device. As the application itself was not specific to CAED, or technology similar to CAED, this article was not included in Figure 4, but collaboration in large spaces does hold potential for CAED implementation. Noor and Aras (2015) used virtual holography to enable multimodal interactions (stylus, 3D gestures and neural input/EEG) for multiuser collaboration intended to be used during design, which would be more accessible and engaging. VR based design systems enabled users to directly create, modify and manipulate virtual models without learning complex protocols and procedures (Igwe et al. 2008). All applications considered some sort of VR environment - stereoscopic projection platform (Bourdot et al. 2010), virtual environment viewed through 3D glasses (Varga et al. 2007), or virtual reality (Zhen-yu and Jian-rong 2005). The inputs varied - voice commands or gesture inputs, using a wand pointing device for direct 3D object manipulations with the dominant hand, while the scene was controlled using a 
glove worn on the users slave hand (Bourdot et al. 2010), the glove with a magnetic tracker embedded, and bi-manual interface (Zhen-yu and Jian-rong 2005).

Some papers discussed frameworks for future implementation (Igwe et al. 2008), others had a strategy for implementing them in commercial software (e.g. CATIA) (Bourdot et al. 2010) to provide a quick simulation or edit solution in VR environment, while some have developed a hand motion language and tested it in commercial modelling software (SolidWorks, SolidEdge and Autodesk) (Varga et al. 2007). Some authors were planning to exhibit realistic material behaviour (Igwe et al. 2008), but none have implemented it (Bourdot et al. 2010; Zhen-yu and Jian-rong 2005).

Due to the early stages of development and lack of full implementation within a commercial software a comparison with a WIMP interface has not yet been achieved for most of the studies (Bourdot et al. 2010). Where the solutions have been implemented practically (Varga et al. (2007), they were evaluated and found that participants with prior experience in CAED scored higher in satisfaction and operability, while all participants produced more original solutions, but reported lower physical comfort.

\subsection{Other interfaces}

Finally a number of reviewed papers had either evaluated the use of existing multimodal interfaces, or developed their own that were unique and did not fit with the main ideas presented in Section 7.5. A short overview of these studies is given here.

Xiangshi and Zhang (2001) believe that the mouse is still useful for accurate modification and location procedures even in multimodal interfaces for CAED systems. They have tested a pen-input tablet, a stylus pen, microphone, a personal computer, mouse, speech recognition in different combinations for effectiveness in a CAED system. The experimental results haven shown that pen, speech and a mouse combination was the best in terms of modification time, total time and subjective evaluation. Sharma et al. (2011) tested speaking and touching, which were chosen as innate abilities to humans. They tested a multi-touch solution (tablet) and multimodal (tablet and voice) solution and found the participants preferred the multimodal interface and have shown shorter task completion time than the multi-touch interface. Stark et al. (2010) explored how designers performed in a VR-cave, free hand sketching using a pen. 2D and 3D conditions were compared. 3D sketches where designers could use the entire 3D space available had proven to be more interactive, faster and the participants stated they felt more stimulated. However no clear benefit in terms of creativity, aesthetics and quality of sketching was noticed. A study was then done where VR sketches were refined in CAED (Rhino), and then the design was reviewed in VR before it was rapid prototyped. Participants preferred the VR environment to classic CAED environment, but these have shown limited usability and lower accuracy, supported rough early modelling, and could not provide a link to detail modelling and refinement. Gao et al. (2000) worked on establishing the approach intended to enable users to intuitively and easily create, modify, manipulate and visualise a solid model by direct 3D manipulations and voice commands, but still remain able to do the 3D manipulations precisely enough so that the system could be used for both early stage design and detailed design. 80 different voice commands and 6DOF mouse were used. Zheng et al. (2001) have decided against voice recognition systems as it was difficult to achieve consistent recognition for the variety of different voices of different users.

These unclassified studies further emphasise how varied the CAED interface development research is and how different the outcomes of the interface evaluations are. Due to the varied nature of the interfaces they are difficult to compare and if any commonality is found across all of them it is that they are all primarily based on one of the existing software or technologies which is then extended by an interface perceived as more intuitive. 


\section{Discussion}

There is an inherent difference in approaches to developing new conceptual CAED systems and new $\mathrm{HCl}$ interfaces. While both focus on avoidance of interruption to the designer's thinking process and fostering creativity, they do it in different ways. CAED software prototypes attempt to understand which requirements are the most important to comply with in order to support the overall design process, give it structure and allow the information created during it to be recorded appropriately. $\mathrm{HCl}$ prototypes focus on the beginning of the process and support uninterrupted data input, and to a lesser degree design refinement and evaluation. None of the approaches in the papers reviewed covered all of the requirements listed in Section 5, they instead focused on a number of requirements they found would be the most beneficial to conceptual design. This is perhaps the biggest shortcoming of all of the solutions reviewed in this paper, and the biggest challenge. While they all report extensive work on successful prototypes, they all focus on a limited number of requirements, solving a limited number of issues, making the solutions unlikely to gain wider acceptance.

As visible from the chronological overview of the CAED systems developed identified in the literature, represented by the category they fall into, in Figure 5, aside from the batch of knowledge based approaches between 2005 and 2014, there are no clearly identifiable trends with regards to timing of the systems developed.

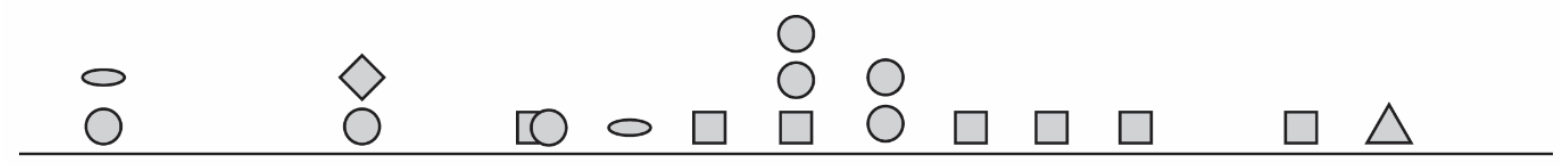

20002001200220032004200520062007200820092010201120122013201420152016

Knowledge based $\bigcirc$ Emulating sketching $\diamond$ Functional

Data capture $\bigcirc$ Feature Based

Figure 5 Chronological overview of the CAED systems development

The categories they fall into are quite diverse, illustrating the breadth of the issues currently present in CAED for conceptual design. They aim to increase creativity, but still enable a designer to reach a concept that is defined enough to provide an input for detailed design without further modifications. Across the different categories, some researchers believe the concepts could be computer generated, but the majority opt for designer generated concepts where a computer is used to support the designer and make the process of designing easier.

Similar to the CAED system development, the CAED interface development cannot be chronologically grouped easily, as visible from Figure 6 . It is clear that the development of versatile touchscreen devices has prompted research into touchscreens as a CAED interface. Haptic interfaces were explored as stand-alone solutions, and then became a part of one of the multimodal gesture based interface groupings, which gain importance in 2006. Aside from a slight preference toward gesture based multimodal solutions since that time, overall it is not apparent that any interface solution type has been particularly dominant in a specific time period. 


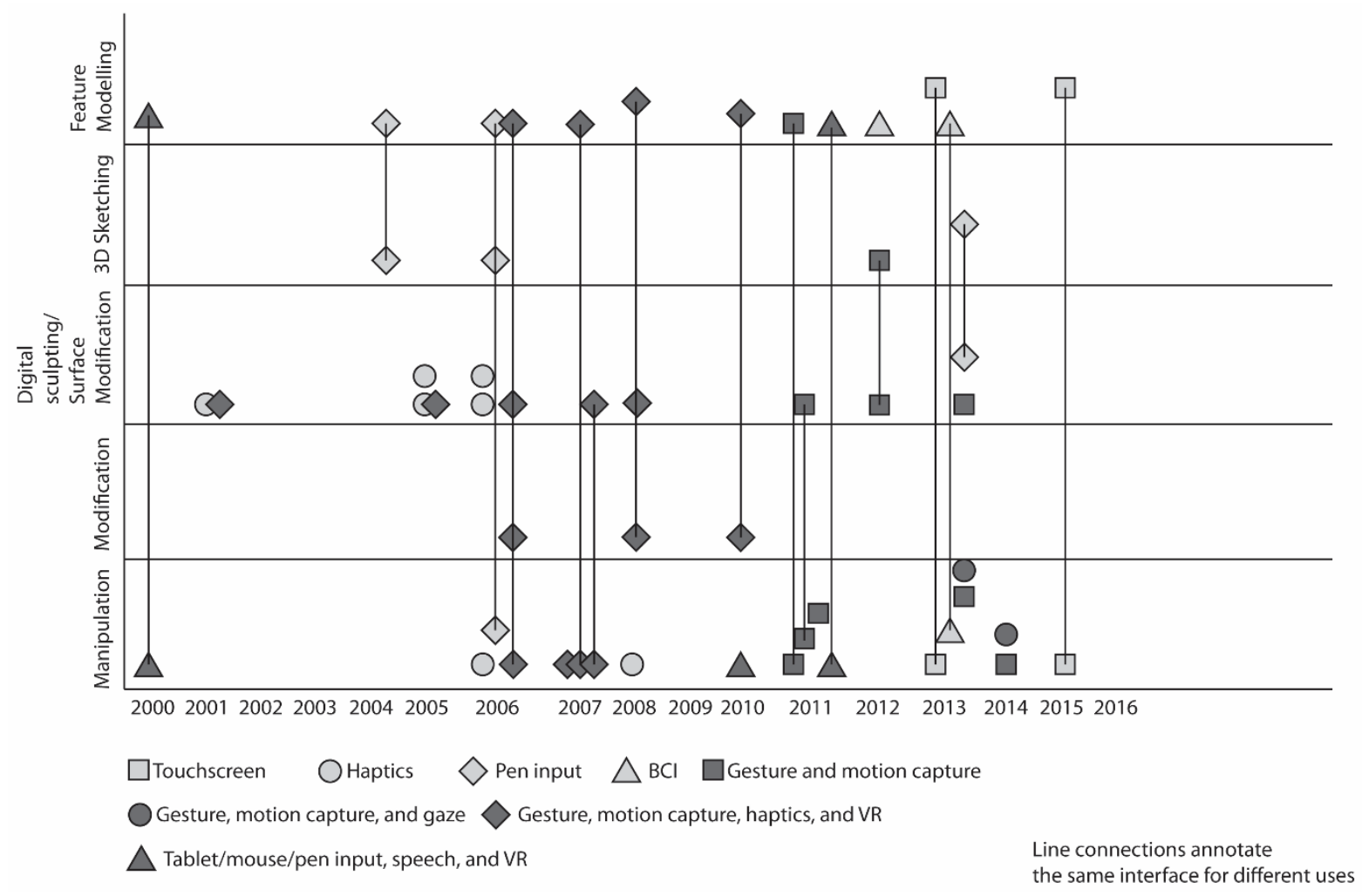

Figure 6 Chronological overview of $\mathrm{HCl}$ research since 2000

None of the CAED systems reviewed had high levels of maturity. While the authors themselves do not classify them with regards to TRL (Technology Readiness Level) scale (Mankins 2009), the most mature ones have reached the stage of technology development or technology demonstration, which corresponds to TRL4, equivalent of a technology being validated in a lab. Comparing the CAED systems reviewed in this articles to the requirements summarized in section 5 , it is visible that the functionalities each of the systems provide only address some of the requirements given in section 5 . For example, key focus in the article by Sivanathan et al. (2015) is to explore capturing and storing metadata providing information about design beyond the standard design records i.e. they partially address recording design intent requirement and building of a knowledge database. Shahin (2008) developed a Database Management System which similarly records the designing process, but also supports it by tracking the designer's progress and automatically finds and suggests similar past designs without interrupting designer's work i.e. addresses provision of knowledge management support to design requirement and assists the human designer without interference with their thinking processes. Therefore, systems were developed with the intention to address a number of very specific requirements, be it knowledge support, emulating sketching, feature design and manipulation, or focus on the function. This meant that even if they were found to successfully solve that issue, they still had required extensive further development in order to become a feasible solution for the commercial conceptual CAED system. Some authors found it hard to compare the newly developed solutions to the existing solutions as the functionalities they offered were very different. Others did not address the issue of measuring and comparing the effectiveness of reviewed CAED systems. Quantifiable testing of effectiveness of newly developed CAED systems in comparison with either commercial CAED systems (in cases where they are used for conceptual design), or analogue traditional conceptual design techniques, would be a valuable measure of success of the newly developed systems. The majority of interfaces developed were likewise at quite an early stage, and even when implemented in commercial CAED systems, they were either not mature enough or only covered specific activities for specific goals. Most intuitive and natural solutions require training, registration of gestures (Song et al. 2014), or calibration. Zheng et al. (2001) believe that the traditional user commands will not be completely eliminated from the existing systems because not all 
tasks are suited to direct manipulation or gestures that are often the basis for new interfaces. Additionally, the sector solutions were developed to include architecture, product design, and engineering design (often automotive design). While these are closely related, they do not necessarily have the identical goals or requirements at the same design stages.

There is a disconnect between conceptual design, currently not fully supported by widely accepted commercial CAED software, and the rest of the design process, such as detailed design, simulation, analysis, manufacturing, which are commonly performed relying on extensive use of CAED/CAM systems. Significant part of motivation for further conceptual CAED development comes from the need to extend the digitalisation of the design process to the conceptual design. A number of CAED systems reviewed in this article discussed the ways to facilitate the connection between conceptual design and the following design stages, or occasionally even considered design propagation throughout all stages using CAED. For example, Müller et al. (2003) and Company et al. (2009) developed different sketch based solutions which are a first step towards a future 3D-sketching tool envisaged to be linked or eventually integrated into existing CAD systems. However, none of the articles have demonstrated a solution which fully addresses the issue.

Out of 39 observed studies on $\mathrm{HCl}$ only 12 have reported the type of experiment performed to test the $\mathrm{HCl}$ and the parameters used to classify participant experience. Majority of studies approximately 20 or less participants, and only perform one experiment to test their prototype. There is very little consistency in the choice of the participants and their backgrounds e.g. both final year engineering students and an engineer with $30+$ years of experience were classed as an experienced CAED software user in two different papers. Additionally, users with different levels of experience were used to test different prototypes. In six of the papers details about the participants were not given, in five the majority of participants were not experienced in CAED software, in four the majority were experienced in CAED software, in one study one participant was experienced in CAED software and the other was not. In three, it was noted that participants did not have prior experience with the tested $\mathrm{HCl}$. Similar tendencies have been noticed in the testing of CAED software prototypes. As they were all low maturity prototypes, they were not tested between experienced and inexperienced groups of users, and no conclusions could have been drawn on how quickly inexperienced users could adopt the new software. In general, for both $\mathrm{HCl}$ and CAED prototypes, the presented results rarely took into consideration the participant's prior experience. The majority of solutions were tested by experienced users, sometimes even those that developed them. This makes it hard to contrast and compare the findings, as different experience levels of the users and different testing parameters might have a significant influence on the findings.

\begin{tabular}{|c|c|c|c|c|c|c|c|c|c|c|}
\hline \multirow[b]{2}{*}{ Data capture } & \multicolumn{3}{|c|}{ CAD software } & \multirow[b]{2}{*}{$\begin{array}{c}\text { Feature } \\
\text { based }\end{array}$} & \multicolumn{5}{|c|}{$\mathrm{HCl}$ prototypes } & \\
\hline & $\begin{array}{c}\text { Knowledge } \\
\text { based }\end{array}$ & Functional & $\begin{array}{l}\text { Emulating } \\
\text { sketching }\end{array}$ & & Manipulation & Modification & $\begin{array}{c}\text { Digital } \\
\text { sculpting }\end{array}$ & 3D sketching & $\begin{array}{l}\text { Feature } \\
\text { modelling }\end{array}$ & \\
\hline : & 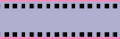 & 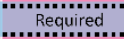 & & & & 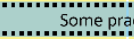 & tice ne............... & achieve & & Declarative knowledge \\
\hline & : & Required & .............. & ................. & & & & & & Procedural knowledge \\
\hline $\begin{array}{l}\text { Seamless } \\
\text { knowledg }\end{array}$ & $\begin{array}{l}\text { strategic } \\
\text { e support }\end{array}$ & $\begin{array}{l}\text { Provides } \\
\text { structured } \\
\text { support } \\
\text { guiding users } \\
\text { through the } \\
\text { process } \\
\text { independently } \\
\text { of their prior } \\
\text { experience }\end{array}$ & $\begin{array}{c}\text { Does not } \\
\text { change current } \\
\text { approach } \\
\text { traditional CAD } \\
\text { systems have } \\
\text { to strategic } \\
\text { knowledge }\end{array}$ & $\begin{array}{l}\text { Provides } \\
\text { structured } \\
\text { support } \\
\text { guiding users } \\
\text { through the } \\
\text { process } \\
\text { independently } \\
\text { of their prior } \\
\text { experience }\end{array}$ & & $\begin{array}{l}\mathrm{HCl} \text { developme } \\
\text { for procedur }\end{array}$ & $\begin{array}{l}\text { th attempts to } \\
\text { land strategi }\end{array}$ & $\begin{array}{l}\text { liminate need } \\
\text { knowledge }\end{array}$ & & Strategic knowledge \\
\hline
\end{tabular}

Figure 7 Types of knowledge and how different CAED/HCl systems address them

Both $\mathrm{HCl}$ and CAED systems reviewed in this article attempt to reduce the need for prior knowledge required to successfully use the systems for conceptual design Figure 7. Procedural knowledge differences between users has been proven to influence the efficiency of CAED software use. $\mathrm{HCl}$ prototypes largely attempt to develop solutions that neutralise the need for specific knowledge, procedural or any other type in order for a successful conceptual design to be developed by the users. The users still need to gain experience in using the specific $\mathrm{HCl}$, and usually with some 
practice they successfully reach good operation levels of knowledge within hours of use. CAED software prototypes acknowledge that prior knowledge of the system is influential, but they similarly aim to lessen its effect. Knowledge based solutions seem to indirectly acknowledge the necessity of software to support task decomposition strategies, as they are attempting to provide support for seamless strategic knowledge support. They also offer support and ideas sourced from databases with collected experiential knowledge. Feature based and functional solutions provide structured support, which helps by guiding the design process independently from the prior experience of the user. Sketching software, similarly to the $\mathrm{HCl}$ approach, tend to attempt to make experience irrelevant, however this is not always possible and they do not fundamentally change the approach traditional CAED systems use. Regardless of the attempt to minimise the effect of prior knowledge on the design process, new CAED software or interfaces still could be significantly influenced by the experience of the user, and the choice of participants for studies used to evaluate them should be carefully thought out, and the influence or lack of influence experience has on CAED systems or $\mathrm{HCl}$ for conceptual design should be clearly demonstrated.

There is no clear direction that researchers have established or believe will become dominant in conceptual CAED systems or $\mathrm{HCl}$ development in the near future. The differences between engineering fields influence the requirements and direction in which conceptual CAED systems are developing. Perhaps the differences are particularly significant here as the parametric design and good links between all design stages are paramount for the successful implementation of any system, and different commercial software dominant in different engineering fields introduce high levels of uncertainty. Observing the $\mathrm{HCl}$ prototypes, haptics seem more mature than other interfaces, but they build on top of conventional CAED, which may arguably limit their effectiveness. Often it is not clear if manipulation or creation are the goal in the new systems, and many interfaces really only do manipulation well, while the creation abilities are limited. This could be because it is currently easier to augment the existing systems with a new manipulation interface than fundamentally change the designing approach. It might indicate the need to consider current CAED systems more carefully when developing new interfaces or software developed to augment them, and likewise consider all the aspects of $\mathrm{HCl}$ that the CAED software systems being developed will employ. $\mathrm{HCls}$ are currently being developed to address the needs of commercially used CAED systems, which do not include conceptual design. This may indicate that either conceptual design requirements should be considered during the $\mathrm{HCl}$ development, or that different interfaces should be developed for the conceptual design stage.

\section{Conclusion}

$\mathrm{HCls}$ largely attempt to incorporate intuitive actions into commercial CAED system interface. Their focus is on better interaction with the system, and they do not consider the nature of the design process as much as the conceptual CAED systems recently developed in academia, do. While clearly used for conceptual design in the context of CAED, due to the nature of the design process which places conceptual design as the first design stage, $\mathrm{HCls}$ often do not focus on conceptual design only, but consider their overall design process, including conceptual design. Perhaps either different $\mathrm{HCls}$ for conceptual design need to be developed, or $\mathrm{HCl}$ development requirements need to be extended to include conceptual design. CAED systems reviewed in this article, on the other hand, focus specifically on the conceptual design stages and ways to support them better. They explore the design process and how it maps onto the CAED software architecture, exploring requirements to support it, challenges in their implementation, and link the unsupported to traditionally computationally well supported design stages. This is not the case in commercially used CAED systems, as they are typically not used for conceptual design, and do not have capabilities to support it well.

Both themes have identified some issues with different levels of expertise of the software users, the definition of what an expert is and how that affects both the design process itself, and the system evaluation. Declarative knowledge is still required for both $\mathrm{HCl}$ and $\mathrm{CAED}$ systems being developed. However, $\mathrm{HCl}$ solutions aim to minimise or even eliminate the need for the procedural and strategic 
knowledge. Novel CAED software prototypes still require procedural knowledge, but tend to provide either seamless support envisaged to reduce the levels of strategic knowledge required to use the systems effectively, or provide structured support which guides the users through the necessary steps.

Both $\mathrm{HCl}$ and conceptual CAED system developments offer solutions that are at the concept level and require further research. CAED system prototypes are arguably at a slightly lower maturity level overall, as the issues they tackle are more complex, and as some of the $\mathrm{HCl}$ solutions, particularly haptic based ones have reached commercial application in other fields such as gaming. One of the key challenges is that commercial CAED system use is established in detailed design and manufacturing, and new solutions need to be incorporated with current commercially used systems, since the industry and the designers are not expected to change their practices easily.

In either of the themes, there is very little consensus on the future steps or a clear path forward. However, there is a high level of agreement on the requirements for the new $\mathrm{HCl}$ and conceptual CAED systems, as well as the main challenges that need to be solved. $\mathrm{HCl}$ requirements primarily focus on intuitive interfaces that allow uninterrupted flow of the design process to take place. New conceptual CAED systems development similarly focuses on supporting the design process by introducing features that enhance it but do not change its inherent structure, and support of continuous development of vague, not entirely defined ideas. While the technological development has helped solve some of the challenges these requirements have introduced, viable solutions for both themes are yet to be found. 


\section{References}

*Alcaide-Marzal J, Diego-Más JA, Asensio-Cuesta S, Piqueras-Fiszman B (2013) An exploratory study on the use of digital sculpting in conceptual product design Design Studies 34:264-284

Asadzadeh P, Kulik L, Tanin E (2012) Gesture recognition using RFID technology Personal and Ubiquitous Computing 16:225-234 doi:10.1145/1620545.1620553. http://dx.doi.org/10.1007/s00779-0110395-z

Bermudez P, Jones S (2016) Early Stage Creative Design Collaboration: A Survey of Current Practice. In: Collaboration in Creative Design. Springer, pp 297-318

Bhavnani SK, Garrett JH, Shaw DS Leading indicators of CAD experience. In: CAAD Futures, 1993. pp 313-334

Bhavnani SK, John BE From sufficient to efficient usage: An analysis of strategic knowledge. In: Proceedings of the ACM SIGCHI Conference on Human factors in computing systems, 1997. ACM, pp 91-98

*Bonnardel N, Zenasni F (2010) The impact of technology on creativity in design: an enhancement? Creativity and innovation management 19:180-191

*Bourdot P, Convard T, Picon F, Ammi M, Touraine D, Vézien J-M (2010) VRCAD integration: Multimodal immersive interaction and advanced haptic paradigms for implicit edition of CAD models Computer-Aided Design 42:445-461

*Brunetti G, Golob B (2000) A feature-based approach towards an integrated product model including conceptual design information Computer-Aided Design 32:877-887

*Bruno F, Giampà F, Muzzupappa M, Rizzuti S A methodology to support designer creativity during the conceptual design phase of industrial products. In: DS 31: Proceedings of ICED 03, the 14th International Conference on Engineering Design, Stockholm, 2003.

Chester I (2007) Teaching for CAD expertise International Journal of Technology and Design Education 17:23-35

${ }^{*}$ Company P, Contero M, Varley P, Aleixos N, Naya F (2009) Computer-aided sketching as a tool to promote innovation in the new product development process Computers in Industry 60:592-603

Dadi GB, Goodrum PM, Taylor TR, Carswell CM (2014) Cognitive workload demands using 2D and 3D spatial engineering information formats Journal of Construction Engineering and Management 140

*Dave D, Chowriappa A, Kesavadas T (2013) Gesture interface for 3d cad modeling using kinect Computer-Aided Design and Applications 10:663669

Dickinson JK, Yu Z, Zeng Y, Antunes H (2005) Pen-tablet as a CAD interface alternative Robotics and Computer-Integrated Manufacturing 21:465474 
Diwakaran RP, Johnson MD (2012) Analyzing the effect of alternative goals and model attributes on CAD model creation and alteration ComputerAided Design 44:343-353

*Dorta T, Perez E, Lesage A (2008) The ideation gap:: hybrid tools, design flow and practice Design Studies 29:121-141

*Esfahani ET, Sundararajan V (2012) Classification of primitive shapes using brain-computer interfaces Computer-Aided Design 44:1011-1019 doi:10.1016/j.cad.2011.04.008

*Evans MA (2005) Rapid prototyping and industrial design practice: can haptic feedback modelling provide the missing tactile link? Rapid Prototyping Journal 11:153-159

Ferguson SM, Olewnik AT, Cormier P (2014) A review of mass customization across marketing, engineering and distribution domains toward development of a process framework Research in Engineering Design 25:11-30

Fiorentino M, Uva AE, Fabiano MD, Monno G (2010) Improving bi-manual 3D input in CAD modelling by part rotation optimisation Computer-Aided Design 42:462-470

*Fuge M, Yumer ME, Orbay G, Kara LB (2012) Conceptual design and modification of freeform surfaces using dual shape representations in augmented reality environments Computer-Aided Design 44:1020-1032

*Gao S, Wan H, Peng Q (2000) An approach to solid modeling in a semiimmersive virtual environment Computers \& Graphics 24:191-202

*Gao Z, Gibson I (2006) Haptic sculpting of multi-resolution B-spline surfaces with shaped tools Computer-aided design 38:661-676

${ }^{*}$ Goel AK, Vattam S, Wiltgen B, Helms M (2012) Cognitive, collaborative, conceptual and creative-four characteristics of the next generation of knowledge-based CAD systems: a study in biologically inspired design Computer-Aided Design 44:879-900

Gomes D, Tzortzopoulos P, Kagioglou M Collaboration through shared understanding in the early design stage. In: 24th Ann. Conf. of the Int'l. Group for Lean Construction, Boston, MA, USA, 20-22 July, 2016. pp 63-72

Haik Y, Shahin T (2010) Engineering Design Process. Cengage Learning, Inc; 2nd edition,

Hanna R, Barber T (2001) An inquiry into computers in design: attitudes before-attitudes after Design Studies 22:255-281

Hartman NW (2009) Defining expertise in the use of constraint-based CAD tools by examining practicing professionals Engineering Design Graphics Journal 69

*Hoeben A, Stappers PJ (2005) Direct talkback in computer supported tools for the conceptual stage of design Knowledge-Based Systems 18:407413

Horváth I Conceptual design: Inside and outside. In: Proceedings of the 2nd International Seminar and Workshop on Engineering Design in Integrated Product, 2000. Citeseer, pp 63-72 
Horváth I, Vroom RW (2015) Ubiquitous computer aided design: A broken promise or a Sleeping Beauty? Computer-Aided Design 59:161-175 doi:http://dx.doi.org/10.1016/j.cad.2014.10.006

*Huang C-CS (2007) Conceptual Modeling Environment (COMOEN). In: Computer-Aided Architectural Design Futures (CAADFutures) 2007. Springer, pp 31-43

*Igwe PC, Knopf GK, Canas R (2008) Developing alternative design concepts in VR environments using volumetric self-organizing feature maps Journal of Intelligent Manufacturing 19:661-675

*Israel JH, Wiese E, Mateescu M, Zöllner C, Stark R (2009) Investigating three-dimensional sketching for early conceptual design-Results from expert discussions and user studies Computers \& Graphics 33:462-473

*IX FD, Qin H, Kaufman A (2001) A novel haptics-based interface and sculpting system for physics-based geometric design Computer-Aided Design 33:403-420

*Jowers I, Prats M, Lim S, McKay A, Garner S, Chase S Supporting reinterpretation in computer-aided conceptual design. In: EUROGRAPHICS Workshop on Sketch-Based Interfaces and Modeling, 2008. pp 151-158

*Kang Y, Kim H, Suzuki H, Han S (2015) Editing 3D models on smart devices Computer-Aided Design 59:229-238

*Kavakli M, Taylor M, Trapeznikov A Designing in virtual reality (DesIRe): a gesture-based interface. In: Proceedings of the 2nd international conference on Digital interactive media in entertainment and arts, 2007. ACM, pp 131-136

*Kim DH, Kim M-J (2006) A new modeling interface for the pen-input displays Computer-Aided Design 38:210-223

Kokotovich V, Purcell T (2000) Mental synthesis and creativity in design: an experimental examination Design studies 21:437-449

*Krish S (2011) A practical generative design method Computer-Aided Design 43:88-100

Kryssanov VV, Tamaki H, Kitamura S (2001) Understanding design fundamentals: how synthesis and analysis drive creativity, resulting in emergence Artificial Intelligence in engineering 15:329-342

*Kyung K-U, Kwon D-S, Yang G-H (2006) A novel interactive mouse system for holistic haptic display in a human-computer interface International Journal of Human-Computer Interaction 20:247-270

Lawson B (2005) Oracles, draughtsmen, and agents: the nature of knowledge and creativity in design and the role of IT Automation in construction 14:383-391

*Lee H, Lim SY, Lee I, Cha J, Cho D-C, Cho S (2013) Multi-modal user interaction method based on gaze tracking and gesture recognition Signal Processing: Image Communication 28:114-126

* Li Y, Wang J, Li X, Zhao W (2007) Design creativity in product innovation The international journal of advanced manufacturing technology 33:213-222 
*Liu X, Dodds G, McCartney J, Hinds B (2005) Manipulation of CAD surface models with haptics based on shape control functions Computer-aided design 37:1447-1458

Mankins JC (2009) Technology readiness assessments: A retrospective Acta Astronautica 65:1216-1223

Markopoulos P, Martens J-B, Malins J, Coninx K, Liapis A (2016) Creativity and collaboration in early design. In: Collaboration in Creative Design. Springer, pp 1-9

*Mayda M, Börklü HR (2014) An integration of TRIZ and the systematic approach of Pahl and Beitz for innovative conceptual design process Journal of the Brazilian Society of Mechanical Sciences and Engineering 36:859-870

Miller GA (1956) The magical number seven, plus or minus two: some limits on our capacity for processing information Psychological review 63:81

*Müller F, Pache M, Lindemann U Digital free-hand sketching in 3D-a tool for early design phases. In: DS 31: Proceedings of ICED 03, the 14th International Conference on Engineering Design, Stockholm, 2003.

Musta'amal AH, Norman E, Hodgson T (2008) CAD as a 'recording'or 'designing'tool: evidence from user behaviours

*Naef M, Payne J Autoeval mkll-interaction design for a VR design review system. In: 3D User Interfaces, 2007. 3DUI'07. IEEE Symposium on, 2007. IEEE,

*Noor AK, Aras R (2015) Potential of multimodal and multiuser interaction with virtual holography Advances in Engineering Software 81:1-6 doi:http://dx.doi.org/10.1016/j.advengsoft.2014.10.004

*Ogawa H, Kosaka K, Suzumori K, Kanda T Development of Active Polyhedron for Physical Human-Machine Interaction and its Application to CAD/CAM Operation. In: Micro-NanoMechatronics and Human Science, 2006 International Symposium on, 2006. IEEE, pp 1-6

*Oh J-Y, Stuerzlinger W, Danahy J Sesame: towards better 3d conceptual design systems. In: Proceedings of the 6th conference on Designing Interactive systems, 2006. ACM, pp 80-89

*Radhakrishnan S, Lin Y, Zeid I, Kamarthi S (2013) Finger-based multitouch interface for performing 3D CAD operations International Journal of Human-Computer Studies 71:261-275

Rahimian FP, Ibrahim R (2011) Impacts of VR 3D sketching on novice designers' spatial cognition in collaborative conceptual architectural design Design Studies 32:255-291

Robertson B, Radcliffe D (2009) Impact of CAD tools on creative problem solving in engineering design Computer-Aided Design 41:136-146

Robertson BF, Walther J, Radcliffe DF (2007) Creativity and the use of CAD tools: Lessons for engineering design education from industry Journal of Mechanical Design 129:753-760

*Rodriguez Esquivel JC, Meneses Viveros A, Perry N (2014) Gestures for interaction between the software CATIA and the human via Microsoft Kinect 
Sener B, Wormald P, Campbell I Towards' virtual clay'modelling-challenges and recommendations: a brief summary of the literature. In: DS 30: Proceedings of DESIGN 2002, the 7th International Design Conference, Dubrovnik, 2002.

*Shahin TM (2008) Computerizing Conceptual Design without Hindering Creativity Computer-Aided Design and Applications 5:548-556

*Shankar SS, Rai R (2014) Human factors study on the usage of $\mathrm{BCl}$ headset for 3D CAD modeling Computer-Aided Design 54:51-55

*Sharma A, Madhvanath S, Shekhawat A, Billinghurst M MozArt: a multimodal interface for conceptual 3D modeling. In: Proceedings of the 13th international conference on multimodal interfaces, 2011. ACM, pp 307310

*Shesh A, Chen B Smartpaper: An interactive and user friendly sketching system. In: Computer Graphics Forum, 2004. vol 3. Wiley Online Library, pp 301-310

*Sivanathan A, Lim T, Ritchie J, Sung R, Kosmadoudi Z, Liu Y (2015) The application of ubiquitous multimodal synchronous data capture in CAD Computer-Aided Design 59:176-191

*Song J, Cho S, Baek S-Y, Lee K, Bang H (2014) GaFinC: Gaze and Finger Control interface for 3D model manipulation in CAD application Computer-Aided Design 46:239-245

*Stark R, Israel J, Wöhler T (2010) Towards hybrid modelling environmentsMerging desktop-CAD and virtual reality-technologies CIRP AnnalsManufacturing Technology 59:179-182

*Tovey M, Owen J (2000) Sketching and direct CAD modelling in automotive design Design Studies 21:569-588

*Tumkor S, Esche SK, Chassapis C Hand Gestures in CAD Systems. In: ASME 2013 International Mechanical Engineering Congress and Exposition, 2013. American Society of Mechanical Engineers, pp V012T013A008-V012T013A008

*Varga E, Horvath I, Rusak Z, Dorozhkin DV, Vance JM (2007) Analysis of the Usability of Hand Motion Language in Shape Conceptualization Guidelines for a Decision Support Method Adapted to NPD Processes

Veisz D, Namouz EZ, Joshi S, Summers JD (2012) Computer-aided design versus sketching: An exploratory case study Artificial Intelligence for Engineering Design, Analysis and Manufacturing 26:317-335

*Verma A, Rai R Creating by Imagining: Use of Natural and Intuitive BCl in 3D CAD Modeling. In: ASME 2013 International Design Engineering Technical Conferences and Computers and Information in Engineering Conference, 2013. American Society of Mechanical Engineers, pp V02AT02A036-V002AT002A036

Verstijnen I, van Leeuwen C, Goldschmidt G, Hamel R, Hennessey J (1998) Sketching and creative discovery Design studies 19:519-546

Vidal R, Mulet E (2006) Thinking about computer systems to support design synthesis Communications of the ACM 49:100-104 
*Wang R, Paris S, Popović J 6D hands: markerless hand-tracking for computer aided design. In: Proceedings of the 24th annual ACM symposium on User interface software and technology, 2011. ACM, pp 549-558

*Xiangshi R, Zhang G (2001) The efficiency of various multimodal input interfaces evaluated in two empirical studies IEICE TRANSACTIONS on Information and Systems 84:1421-1426

*Ye J, Campbell R, Page T, Badni K (2006) An investigation into the implementation of virtual reality technologies in support of conceptual design Design Studies 27:77-97

*Zhen-yu L, Jian-rong T (2005) Interactive sculpting of product shape based on constraint manipulation in virtual environment Journal of Zhejiang University SCIENCE A 6:733-740

*Zheng J, Chan K, Gibson I (2001) Desktop virtual reality interface for computer aided conceptual design using geometric techniques Journal of Engineering Design 12:309-329

*Zhong K, Kang J, Qin S, Wang H Rapid 3d conceptual design based on hand gesture. In: Advanced Computer Control (ICACC), 2011 3rd International Conference on, 2011. IEEE, pp 192-197

*Zhu W (2008) A methodology for building up an infrastructure of haptically enhanced computer-aided design systems Journal of Computing and Information Science in Engineering 8:041004 


\section{Glossary}

6DOF 6 Degrees of Freedom

$\mathrm{BCl}$ Brain Computer Interfaces

CAED Computer Aided Design

CAM Computer Aided Manufacturing

EEG Electroencephalography

EMG Electromyography

FBS Function -Behaviour-Structure

GUI Graphical User Interface

$\mathrm{HCl}$ Human Computer Interface

RFID Radio-frequency identification

TRIZ Design innovation tool

TRL Technology Readiness Level

VR Virtual Reality

WIMP Windows Icon Menu Pointer 